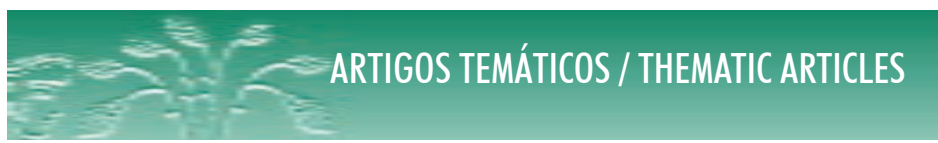

DOI: http://dx.doi.org/10.12957/demetra.2014.10346

\title{
Qualidade de vida de pacientes com anorexia e bulimia nervosa
}

\section{Quality of life of patients with anorexia and bulimia nervosa}

\author{
Erika Arantes de Oliveira-Cardoso \\ Bruna Vieira Von Zuben' \\ Manoel Antônio dos Santos' \\ 'Departamento de Psicologia, Faculdade de
Filosofia, Ciências e Letras de Ribeirão Preto.
Universidade de São Paulo. Ribeirão Preto,
SP, Brasil.
}

Correspondência / Correspondence

Érika Arantes de Oliveira Cardoso

E-mail: erikaao@ffclrp.usp.br

\section{Resumo}

Anorexia e bulimia nervosa constituem os tipos principais de perturbações graves do comportamento alimentar, que acometem sobretudo adolescentes e jovens adultos do sexo feminino. Esses quadros assemelham-se quanto à acentuada distorção da imagem corporal, medo mórbido de engordar, preocupação excessiva em relação à alimentação e desejo não realista e persistente de emagrecimento. Este estudo teve por objetivo ampliar o conhecimento acerca da singularidade do funcionamento psicodinâmico e dos fenômenos psicopatológicos a eles subjacentes. Foram avaliados seis participantes em tratamento num serviço multidisciplinar especializado. Os instrumentos utilizados para a coleta de dados foram: Teste de Atitudes Alimentares (EAT-26) e Questionário Genérico de Avaliação de Qualidade de Vida (SF-36). Os dados obtidos indicam que, na média geral, os componentes mentais da qualidade de vida das participantes estão mais comprometidos do que os componentes físicos. Em relação ao domínio mais favorecido, destaca-se o "Estado Geral de Saúde", seguido por "Dor" e "Capacidade Funcional". Já o domínio "Saúde Mental" se apresenta como o mais prejudicado. Os resultados mostram que, de modo geral, a qualidade de vida dos participantes encontra-se preservada, à exceção dos domínios "Saúde Mental" e "Aspectos Emocionais". Conclui-se que o construto "Qualidade de Vida" é um indicador importante a ser considerado pela equipe multidisciplinar.

Palavras-chave: Bulimia Nervosa. Anorexia Nervosa. Qualidade de Vida. Saúde Mental. 


\section{Abstract}

Anorexia nervosa and bulimia nervosa are the main types of serious eating disorders, particularly in adolescents and young female adults. They are similar concerning the sharp distortion of body image, morbid fear of gaining weight, concern with food and constant unrealistic desire for weight loss. This study aimed to assess the implication of eating disorders on the quality of life of patients with anorexia and bulimia nervosa. Six participants under treatment of eating disorders in a multidisciplinary team were evaluated. The instruments Eating Attitudes Test (EAT- 26) and the Medical Outcomes Study 36-Item Short-Form Health Survey (SF-36) were used for data collection. The data indicate that the mental components of quality of life of participants are more impaired than the physical components. The "General Health Perceptions" were the most favored domain, followed by "Bodily Pain" and "Physical Functioning". "General Mental Health" is the most impaired domain. The results show that participants' quality of life is preserved, except for "General Mental Health" (psychological distress and well-being) and "Role Limitation Due to Emotional Problems". "Quality of live" is an indicator to be considered, especially by the multidisciplinary team.

Key words: Bulimia Nervosa. Anorexia Nervosa. Quality of life. Mental Health.

\section{Introdução}

Transtornos alimentares (TAs) são quadros psiquiátricos que afetam principalmente jovens e adultos do sexo feminino, acarretando sérios prejuízos biopsicossociais e com elevada morbidade e mortalidade. Anorexia nervosa (AN) e bulimia nervosa (BN) constituem os dois principais tipos de transtornos descritos, nos quais o peso e o formato corporal exercem importante influência na determinação da autoestima dos pacientes. ${ }^{1}$

A AN caracteriza-se, essencialmente, por perda de peso intensa, à custa de rígidas dietas autoimpostas na busca desenfreada pela magreza, distorção da imagem corporal e amenorreia. ${ }^{2}$ Por sua vez, a BN caracteriza-se por ingestão de grande quantidade de alimentos de maneira muito rápida e com sensação de perda de controle, os chamados “episódios bulímicos", acompanhados de métodos compensatórios inadequados para o controle de peso, como vômitos autoinduzidos, uso de medicamentos (diuréticos, laxantes, inibidores de apetite), dietas e exercícios físicos extenuantes, abuso de cafeína ou uso de cocaína. ${ }^{2}$ 
A prevalência da AN é de 0,5 a 3,7\% e da BN, de 1,1\% a 4,2\%, dependendo dos critérios usados para definição dos transtornos. ${ }^{3} \mathrm{O}$ maior acometimento de transtornos alimentares na população mundial ocorre em pacientes jovens, de idade entre 12 e 25 anos, e mulheres (90\% dos casos). Já o Brasil não conta com dados estatísticos para transtornos alimentares. A baixa predominância na população e a subnotificação dos casos figuram como dificuldades para estabelecer uma prevalência correta dos TAs. ${ }^{3}$

O comportamento alimentar, além do ato de comer, também envolve a experiência de estímulos internos e externos, considerando fatores orgânicos, psicológicos e sociais. ${ }^{4}$ Assim, o ato de comer transcende o valor nutritivo e as características sensoriais do alimento, tendo em vista a presença de motivações ocultas relacionadas às carências psicológicas e às vivências emotivas e conflituosas, que independem da fome. ${ }^{5}$

Diversos fatores contribuem para a predisposição, instalação e manutenção dos sintomas dos TAs. Dessa forma, é reconhecida a influência da dinâmica familiar, do meio sociocultural e do funcionamento da personalidade do indivíduo como componentes determinantes do curso desses transtornos. ${ }^{6,7}$ Nesse contexto, a dinâmica familiar é vista como importante fator de risco para o desenvolvimento de AN e BN, contribuindo para a construção de um discurso social patologizante, tendo em vista que a atribuição de culpa, muitas vezes reservada aos familiares, contribui para que pais e mães sintam-se ainda mais impotentes ante o caráter insólito dos sintomas de seus filhos. ${ }^{8}$ Assim,os TAs costumam desorganizar o grupo familiar, complicando seu funcionamento.

Em relação ao ambiente sociocultural, na sociedade ocidental a importância conferida à aparência física e ao padrão valorizado de beleza, sobretudo nas últimas décadas, tem contribuído para uma autêntica "ditadura dos corpos esbeltos". Os meios de comunicação de massa tendem a perpetuar esse ideal de magreza, uma vez que reforçam o estigma em relação ao corpo que difere de tais padrões, o que pode influenciar o desenvolvimento e a manutenção dos sintomas de TAs. ${ }^{9}$

Por fim, em relação aos aspectos emocionais, a literatura aponta para um funcionamento afetivo-emocional bastante perturbado no contexto desses quadros psicopatológicos demarcados pela perturbação no comportamento alimentar, emergindo sentimentos de medo, culpa, vergonha e ansiedade na relação com o alimento. ${ }^{9}$

Nas últimas cinco décadas, a importância da avaliação da qualidade de vida esteve em ascensão, priorizando-se instrumentos mais curtos e mais fáceis de usar e entender. A definição de qualidade de vida adotada pela Organização Mundial da Saúde (OMS) é composta por três características: subjetividade (ênfase na percepção subjetiva, ou seja, do próprio indivíduo), multidimensionalidade (considerando aspectos físicos, psicológicos e sociais) e bipolaridade (dimensões positivas e negativas). Desse modo, valoriza-se a noção do bem-estar subjetivo, sem ignorar a influência de fatores externos, como trabalho e relações sociais, nessa percepção. ${ }^{10}$ 
Um dos instrumentos mais utilizados em todo o mundo para avaliar a qualidade de vida é o questionário Medical Outcomes Study 36-Item Short-Form Health Survey (SF-36), criado a partir da necessidade de se ter um instrumento padronizado que abordasse conceitos gerais de saúde, não específicos para qualquer condição médica, de modo a serem compreensíveis, de fácil manuseio e utilização, e psicometricamente adequados. Já o conceito de qualidade de vida abordado nesse instrumento, é dado pela qualidade de saúde relacionada à vida, que ressalta o impacto que a prevenção e o tratamento de uma enfermidade têm sobre o "valor de se estar vivo". ${ }^{11}$

O SF-36 é útil para comparar populações gerais e amostrais, cotejando o impacto relativo de doenças, de maneira a diferenciar os benefícios produzidos por diferentes tratamentos. ${ }^{11} \mathrm{E}$ composto de 36 questões, que fornecem pontuações nos seguintes domínios de qualidade de vida: a) Capacidade Funcional, avaliada a partir do desempenho de atividades diárias, como aptidão para cuidar de si, vestir-se, tomar banho, subir escadas; b) Limitação por Aspectos Físicos, indicada pelo impacto da saúde física no desempenho das atividades diárias e/ou profissionais; c) Dor, conforme o nível de dor e o impacto no desempenho das atividades diárias e/ou profissionais; d) Estado Geral de Saúde, entendido pela percepção subjetiva do estado geral de saúde; e) Vitalidade, assinalada pela disposição dos pacientes em realizar tarefas do cotidiano; f) Aspectos Sociais, pelo reflexo da condição de saúde nas atividades sociais; g) Aspectos Emocionais, pelos reflexos das condições emocionais no desempenho das atividades diárias e/ou profissionais; e h) Saúde Mental, pela escala de humor e bem-estar. ${ }^{12}$

Revisão sistemática da literatura, ${ }^{13}$ focalizando a qualidade de vida de pacientes com TAs, apontou maior comprometimento quando comparada à qualidade de vida de sujeitos sem psicopatologias alimentares ou da população normativa. Os resultados obtidos no referido estudo sugerem que a qualidade de vida de sujeitos que apresentam quadros de AN e BN parece estar associada a maiores prejuízos emocionais e sociais em relação aos aspectos físicos, embora o aumento da atividade física possa ser eventualmente uma manifestação psicopatológica (mais uma tática utilizada para alcançar o emagrecimento), o que torna tal dado duvidoso. ${ }^{13}$

Ainda em relação ao estudo de revisão, o aspecto mais afetado da qualidade de vida em pacientes com TAs foi o social, o que sugere a necessidade de se considerar os aspectos clínicos e psiquiátricos, conjuntamente ao suporte social e envolvimento familiar, no curso do tratamento e planejamento clínico de pacientes com TAs. A presença de comorbidades, sintomatologia depressiva ou ansiosa e a gravidade do quadro alimentar mostraram-se correlacionadas a níveis mais elevados de prejuízo. ${ }^{13}$

Outro dado da revisão aponta que a subescala "Capacidade Funcional" do instrumento SF-36, que envolve a realização de atividades básicas e movimentação física, apresenta pouco ou nenhum prejuízo à qualidade de vida de sujeitos com TAs. Isso indica que, quanto maior a hiperatividade, 
maior o nível de qualidade de vida nesse domínio. Entretanto, a melhor qualidade de vida refletida pela Capacidade Funcional elevada pode estar relacionada a maior nível de psicopatologia alimentar, e não o contrário, como se desejaria aferir. ${ }^{13}$ Dessa forma,

[...] dado que a avaliação da qualidade de vida é necessariamente subjetiva, talvez não seja o modo de avaliação da qualidade de vida a questão a ser resolvida, mas a própria complexidade dos transtornos alimentares. Os resultados até o momento permitem refletir sobre formas de contornar as dificuldades inerentes à avaliação da qualidade de vida nesse grupo de pacientes, mas não eliminá-la, e tampouco concluir qual é a melhor forma de fazê-lo (p. 446).

Assim, frente à complexidade dos quadros de TAs, o profissional de saúde necessita conhecer a singularidade e o funcionamento dos fenômenos psicopatológicos subjacentes a esses quadros, bem como suas implicações na clínica, para que possa traçar estratégias mais efetivas de intervenção. ${ }^{6,9}$

Além do SF-36 e sua possível aplicação no contexto da avaliação das implicações dos TAs, outro instrumento utilizado essencialmente nesse universo é o EAT, que tem como objetivo aferir comportamento de risco e sintomas de TAs em pacientes ou estudos populacionais. Pela facilidade de sua aplicação, eficiência, economia, e também porque não há necessidade de treinamento para a administração, o EAT é provavelmente o mais utilizado de todos os instrumentos de autopreenchimento na área dos TAs. Sua versão mais abreviada com 26 questões, o EAT-26, não faz diagnóstico do transtorno, mas detecta casos clínicos em população de alto risco e identifica indivíduos com preocupações anômalas com relação a peso e alimentação. ${ }^{14}$

Tendo em vista a importância de estudos sobre qualidade de vida nos TAs, considerando sua elevada incidência na sociedade contemporânea, bem como os índices de morbidade e mortalidade relacionados a seu diagnóstico, este estudo teve por objetivo favorecer o contato com o universo complexo dos TAs, ampliando o conhecimento acerca da singularidade do funcionamento psicodinâmico e dos fenômenos psicopatológicos a eles subjacentes, por meio dos instrumentos SF-36 e EAT-26.

\section{Contexto do estudo}

O estudo foi realizado em um Ambulatório de Nutrologia de um hospital público do interior de São Paulo, com uma equipe composta por médicos nutrólogos, nutricionistas, psiquiatras, psicólogos e estudantes-estagiários de Psicologia, além de enfermeiros e terapeutas ocupacionais nos casos de internação. Esses profissionais atuam, em sua maioria, voluntariamente e se inserem numa instituição macro-hospitalar pública, de natureza acadêmica, atendendo a diferentes classes sociais devido a sua inserção no Sistema Único de Saúde (SUS). 
As modalidades de assistência oferecidas, em nível ambulatorial, com funcionamento semanal, compreendem: atendimentos clínicos individuais realizados por nutrólogos e nutricionistas; atendimentos psiquiátricos; atendimentos psicoterápicos individuais para pacientes e alguns familiares; psicodiagnóstico; grupo de orientação médico-nutricional; e grupo de apoio psicológico aos familiares. Em média, são atendidos de seis a oito pacientes em cada retorno.

\section{Método}

Estudo descritivo exploratório, de abordagem quantitativa, com amostra de conveniência composta pelos pacientes que iniciaram o atendimento durante o segundo semestre de 2012. Foram avaliados seis participantes, quatro mulheres e dois homens, em tratamento para TA numa equipe multidisciplinar especializada.

Adotou-se como princípio básico o respeito aos voluntários e à instituição, sendo o projeto aprovado pelo Comitê de Ética em Pesquisa do Hospital das Clínicas da Faculdade de Medicina de Ribeirão Preto, de acordo com o processo número 3749/2009. Os participantes foram informados dos objetivos da pesquisa e a coleta foi realizada após leitura e assinatura do Termo de Consentimento Livre e Esclarecido.

As avaliações foram realizadas individualmente, em sala reservada e com condições físicas e de conforto adequadas, junto ao Hospital das Clínicas da Faculdade de Medicina de Ribeirão Preto.

Os instrumentos utilizados para a coleta de dados foram o Teste de Atitudes Alimentares, EAT-26 ${ }^{15}$ e o Questionário Genérico de Avaliação de Qualidade de Vida, SF-36, ${ }^{16}$ aplicados em situação face a face nos retornos ambulatoriais.

Os resultados foram analisados de acordo com as recomendações específicas da literatura, sendo que no EAT uma pontuação acima de 21 é indicativa de TA. No SF-36, os resultados são transformados em um valor que varia de 0 a 100, sendo que, quanto mais próximo do 100, mais preservado está aquele aspecto da qualidade de vida. 


\section{Resultados}

A figura 1 mostra a distribuição dos escores médios obtidos nos domínios da qualidade de vida avaliados pelo SF-36.

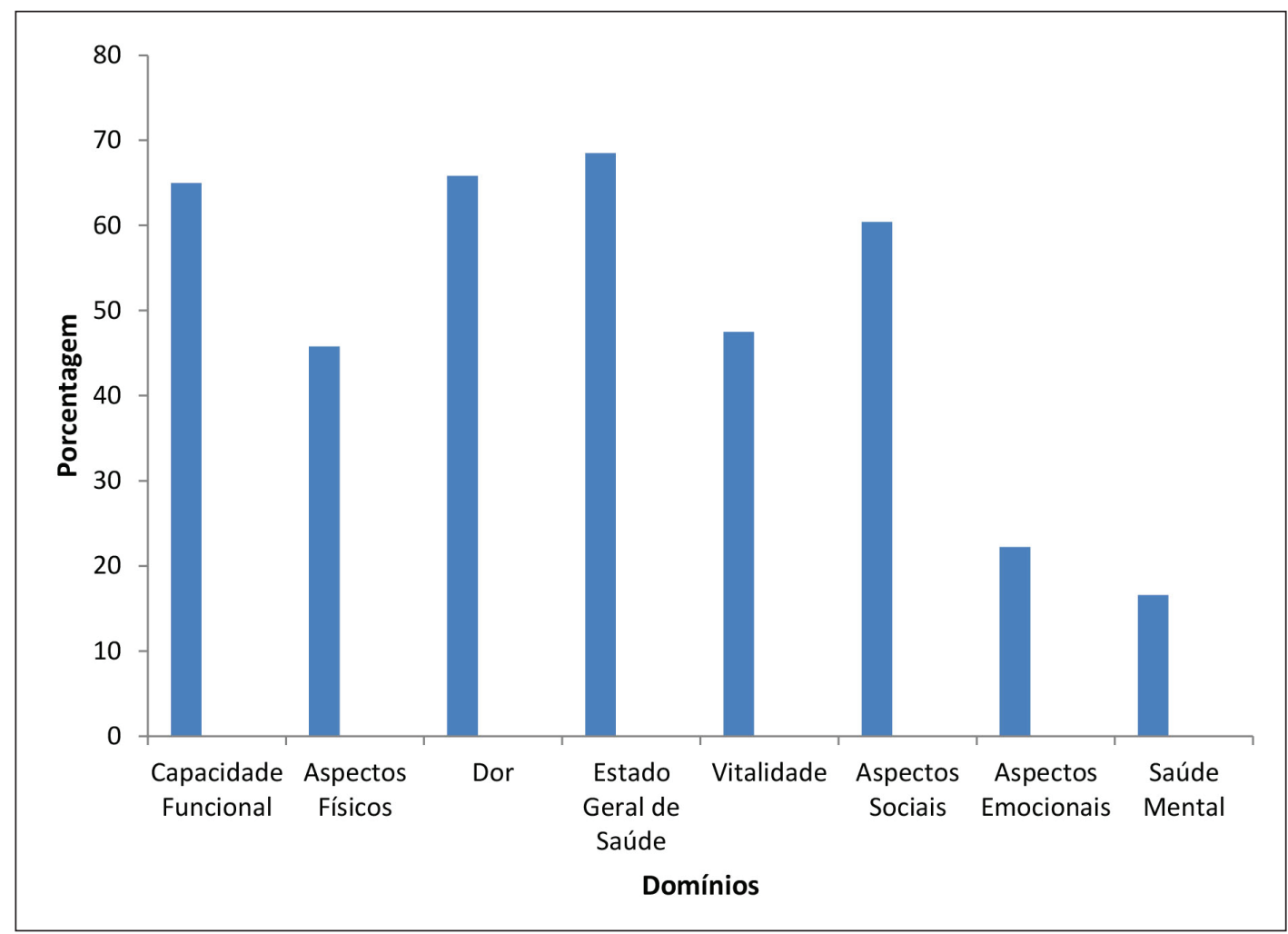

Figura 1. Distribuição média dos escores obtidos nos oito domínios avaliados pelo questionário SF-36. 
A figura 2 mostra as pontuações de cada participante no Teste de Atitudes Alimentares (EAT-26).

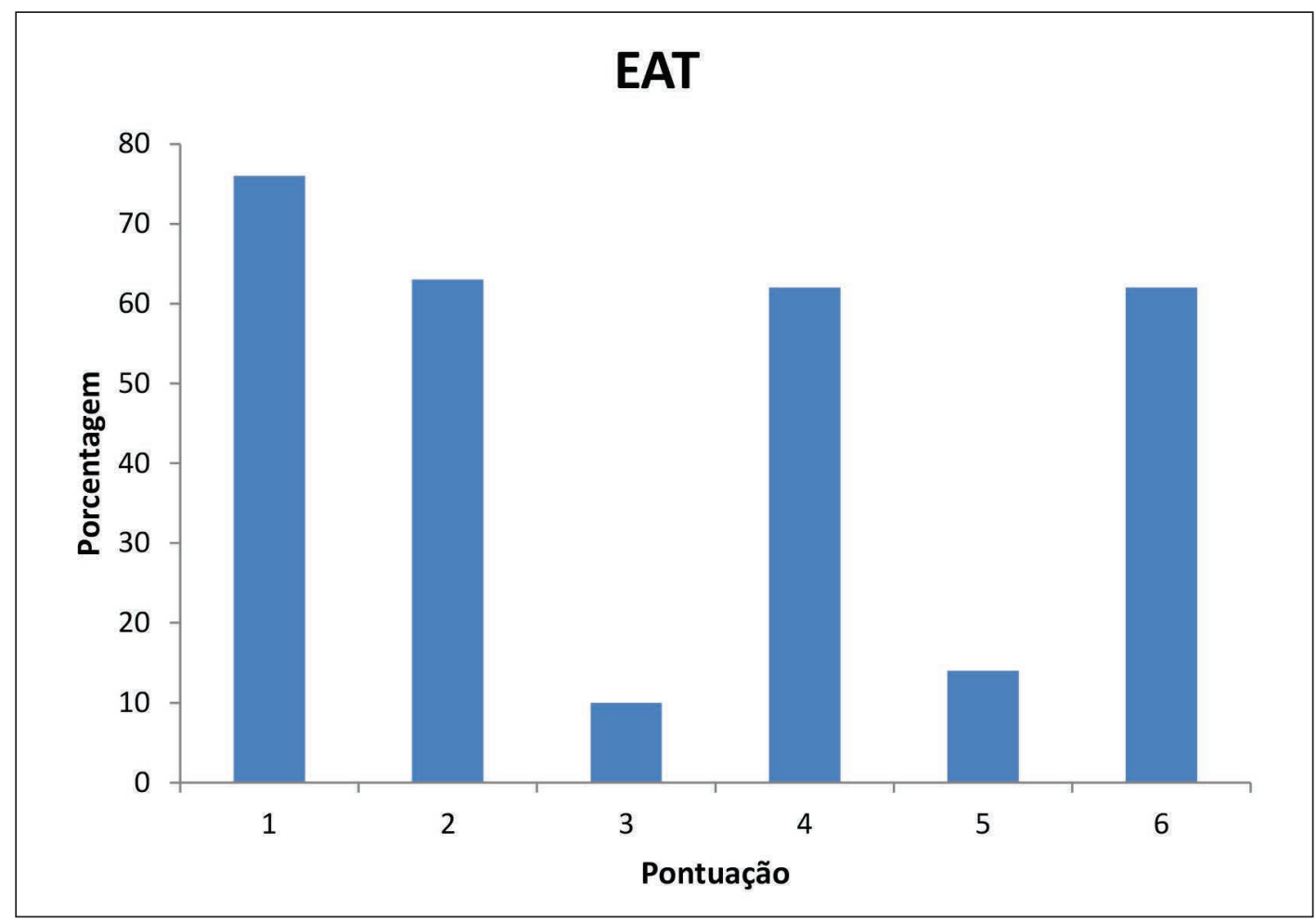

Figura 2. Distribuição das pontuações obtidas no EAT

\section{Discussão}

Em relação aos dados avaliados pelo EAT-26, os resultados obtidos confirmam a presença de sintomas de TAs em quatro participantes. A pontuação média obtida $(47,8 ; \mathrm{DP}=28,3)$ foi muito superior ao ponto de corte da escala (21) nos participantes 1, 2, 4 e 6 . Os participantes 3 e 5 obtiveram escores abaixo do ponto de corte (respectivamente, 10 e 14 pontos), o que pode ser explicado pelo fato de estarem em tratamento. 
Já os dados obtidos por meio do SF-36 indicam que, na média geral, os componentes mentais da qualidade de vida dos participantes estão mais comprometidos do que os componentes físicos, tendo em vista, sobretudo, os baixos escores nos domínios "Saúde Mental" (média $=16,5, \mathrm{DP}=27,6$ ) e "Aspectos Emocionais" (média = 22,2, DP = 40,35), corroborando estudo de Tirico, Stefano e Blay (2010). Os escores obtidos em "Saúde Mental" variaram entre 16 e 44 e, em "Aspectos Emocionais", entre 0 e 100. Pode-se afirmar que, devido a suas condições emocionais, os participantes mostram baixo desempenho em suas atividades diárias e/ou profissionais. Também apresentaram baixa pontuação no domínio relativo ao estado de humor e bem-estar psicológico.

Os escores médios obtidos nos domínios "Aspectos Físicos" (média $=45,8, \mathrm{DP}=51,0$ ) e "Vitalidade" (média $=47,5, \mathrm{DP}=42,8$ ) encontram-se abaixo de 50, o que sugere tendência a rebaixamento. Esse resultado é compatível com a descrição clínica de pacientes com TAs. Os escores obtidos em "Aspectos Físicos" e "Vitalidade" variaram entre 0 e 100. Esses resultados são corroborados pelas observações da prática clínica dos TAs. Pacientes com graus severos de desnutrição se queixam de complicações físicas, fraqueza geral, suscetibilidade à fadiga e perda de energia vital.

Em relação aos domínios mais favorecidos, destaca-se o "Estado Geral de Saúde" (média = 68,5, DP $=24,7$ ), seguido bem de perto de "Dor" (média $=65,8, \mathrm{DP}=38,9$ ) e "Capacidade Funcional" (média $=65,0, \mathrm{DP}=34,3$ ). Esses resultados indicam que os participantes se autoavaliaram como tendo condições gerais de saúde relativamente boas (em comparação com os demais domínios avaliados), assim como ausência de dor e funcionalidade. Este último domínio é expresso a partir do desempenho de atividades diárias, como aptidão para cuidar de si, vestir-se, tomar banho, ou pela movimentação física.

O escore relativamente elevado da "Capacidade Funcional" também corrobora a literatura, ${ }^{13}$ e pode indicar uma manifestação psicopatológica própria do transtorno, uma vez que os indivíduos diagnosticados muitas vezes se submetem a exercícios físicos extenuantes ou fazem rigorosas dietas restritivas, em busca de um corpo cada vez mais magro, por influência da grave distorção da imagem corporal e também de padrões culturais/estéticos difundidos pelos veículos de comunicação em massa.

Como apontado, o domínio "Saúde Mental” apresentou-se como o domínio mais comprometido dos participantes do presente estudo. Os quadros psicopatológicos característicos da AN e BN são marcados por graves perturbações no comportamento alimentar, esboçadas pelo medo mórbido de engordar, pela busca de obter alívio da ansiedade por meio da comida (episódios bulímicos ou de compulsão alimentar, binge eating) e pelo sentimento de culpa de tê-la ingerido, culminando tipicamente em esforços para eliminar os alimentos consumidos. ${ }^{9}$ 
Por fim, em relação aos "Aspectos Sociais" (Média $=60,4, \mathrm{DP}=39,2$ ), os resultados não apresentaram escores tão baixos como o reportado pela literatura. ${ }^{13}$ Desse modo, o apoio social e o envolvimento familiar, nesses pacientes que se encontram em tratamento, parecem estar, de certo modo, preservados.

Ao comparar os resultados obtidos nos instrumentos aplicados, observa-se que os dois participantes que obtiveram menor pontuação no EAT apresentaram melhor qualidade de vida, com ao menos seis dos oitos componentes do SF-36 preservados. Esse dado sugere que os sintomas repercutem na qualidade de vida, contribuindo para sua depreciação. No entanto, considerando as limitações do presente estudo e seu delineamento, os resultados não fornecem apoio empírico a essa hipótese, que deverá ser investigada por estudos futuros.

\section{Conclusões}

Observa-se que, em geral, a qualidade de vida dos participantes encontra-se relativamente preservada, à exceção de "Aspectos Emocionais" e "Saúde Mental”. Esse dado deve ser levado em consideração pela equipe multiprofissional, uma vez que pode ser um indicador de que o maior comprometimento nos aspectos psicológicos aponta para áreas de vulnerabilidade que precisam ser contempladas no plano terapêutico. Abordagens psicoterapêuticas de apoio podem ser indicadas, dentro de uma estratégia que visa ao fortalecimento de defesas psicológicas mais amadurecidas, que possam se contrapor às defesas mais arcaicas e desadaptativas.

A psicoterapia combinada - individual e em grupo (ou psicoterapia familiar) - pode ser um excelente recurso para se alcançar um padrão de funcionamento psicológico mais integrado e maximizar os benefícios terapêuticos advindos das demais estratégias utilizadas, como a reabilitação nutricional e o acompanhamento medicamentoso. ${ }^{17}$

Além disso, os resultados apontam também para a importância de se contar com uma equipe multiprofissional altamente qualificada, que atenda às diferentes necessidades e aspectos da qualidade de vida. O tratamento dos TAs requer a inclusão de profissionais qualificados no cuidado dos componentes mentais, que frequentemente se apresentam comprometidos nesses pacientes, mais até do que os componentes físicos. 
Uma das limitações do estudo é o tamanho reduzido da amostra. A maior parte dos escores apresentou grande amplitude de variação, o que distorce o significado da média. Isso impõe a necessidade de investigar amostras maiores. $\mathrm{O}$ instrumento utilizado para investigar a qualidade de vida é genérico, o que sugere a necessidade de complementar os dados por meio de instrumentos específicos. O estudo também se beneficiaria com a inclusão de outro instrumento para identificar os sintomas de TAs, como entrevista clínica padronizada, a fim de captar as nuances dinâmicas dos quadros clínicos.

\section{Referências}

1. Hay PJ. Understanding bulimia. Australian Family Physician 2007; 36(9):708-731.

2. Abreu CN, Cangelli Filho R. Anorexia nervosa e bulimia nervos: abordagem cognitivo-construtivista da psicoterapia. Psicologia: Teoria e Prática. São Paulo, 2005; 7(1):153-165.

3. American Psychiatric Association Practice Guidelines. Practice guideline for the treatment of patients with eating disorders. Am. J. Psychiatry 2000; 157(Supl.1):1-39.

4. Castillo MCC, Acharán X, Alvarez P, Bustos MCP. Apetito y nutrición. Revista Chilena Pediátrica 1990; 61(6):346-353.

5. Dallerra S, Sorrentino N. A psicodieta: por que comemos, por que engordamos, como parar. São Paulo: Paulus; 1997.

6. Oliveira EA, Santos MA. Perfil psicológico de pacientes com anorexia e bulimia nervosas: a ótica do psicodiagnóstico. Medicina (Ribeirão Preto), 2006; 39(3):353-360.

7. Dupont ME, Corcos M. Psychopathology in eating disorders: new trends. La Revue du Praticien 2008; 58(2):141-149.

8. Dodge E, Matthew H, Dare C. Family therapy for bulimia nervosa in adolescents: an exploratory study. Journal of Family Therapy 2005; 17(1):59-77.

9. Oliveira-Cardoso EA, Santos MA. Avaliação psicológica de pacientes com anorexia e bulimia nervosas: indicadores do Método de Rorschach. Fractal: Revista de Psicologia 2012, 24(1):159-174.

10. Giacomini CH. Bem-estar subjetivo: em busca da qualidade de vida. Temas em Psicologia 2004; 12(1):43-50.

11. Cruz LN, Fleck MPA, Oliveira MR, Camey SA, Hoffmann JF, Bagattini AM, et al. Health-related quality of life in Brazil: normative data for the SF-36 in a general population sample in the south of the country. Ciência Saúde Coletiva 2013; 18(7):1911-1921.

12. Cattai GBP, Rocha FA, Nardo Junior N, Pimentel GGA. Qualidade de vida em pacientes com insuficiência renal. Ciências, Cuidado \& Saúde 2007; 6(2):460-467. 
13. Tirico PP, Stefano CS, Blay LS. Qualidade de vida e transtornos alimentares: uma revisão sistemática. Caderno de Saúde Pública 2010; 26(3):431-449.

14. Alvarenga MS, Scagliusi FB, Philippi ST. Comportamento de risco para transtorno alimentar em universitárias brasileiras. Revista de Psiquiatria Clínica 2011; 38(1):3-7.

15. Bighetti F. Tradução e validação do Eating Attitudes Test (EAT-26) em adolescentes do sexo feminino na cidade de Ribeirão Preto - SP [dissertação]. Ribeirão Preto: Universidade de São Paulo; 2003.

16. Ciconelli RM, Ferraz MS, Santos W, Meinão I, Quaresma MR. Tradução para a língua portuguesa e validação do questionário genérico de qualidade de vida SF-36. Revista Brasileira de Reumatologia 1999; 39(3):143-150.

17. Santos MA. Sofrimento e esperança: grupo de pacientes com anorexia e bulimia nervosas. Medicina (Ribeirão Preto) 2006; 39(3):286-401.

Recebido: 07/4/2014

Revisado: 29/5/2014

Aprovado: 07/7/2014 